\title{
ESTUDO PRELIMINAR DA DINÂMICA DE AQUECIMENTO EM CONCENTRADORES SOLARES
}

\author{
L. A. ANDRADE, M. A. S. BARROZO e L. G. M. VIEIRA \\ Universidade Federal de Uberlândia, Faculdade de Engenharia Química \\ E-mail para contato: luizgustavo@feq.ufu.br
}

\begin{abstract}
RESUMO - A luz solar é uma energia renovável, limpa e abundante, podendo ser utilizada como fonte térmica mediante à captação em concentradores solares. Isto posto, este trabalho teve como principal objetivo prever o comportamento temporal da temperatura de um corpo inserido no ponto focal de um concentrador solar tipo paraboloide de revolução. Para tanto, simulações computacionais foram realizadas mediante a manipulação do diâmetro do coletor solar, da massa sólida a ser aquecida e da taxa de irradiação solar, organizadas sob a forma de um Planejamento Composto Central (PCC). De acordo com os principais resultados simulados, foi possível constatar que temperaturas de até $1284^{\circ} \mathrm{C}$ puderam ser facilmente alcançadas em períodos de tempo relativamente baixos. Tais resultados preliminares indicaram que os concentradores solares do tipo parabolóide de revolução podem ser uma interessante alternativa para prover a energia térmica requerida em sistemas de mero aquecimento ou reacionais (pirólise).
\end{abstract}

\section{INTRODUÇÃO}

Dentre as fontes de energias renováveis, uma que vem ganhando visibilidade é a energia solar (Narbel et al., 2011). A energia solar tem experimentado nas últimas décadas um grande desenvolvimento tecnológico haja vista que, no início as tecnologias solares, consistiam-se em células fotovoltaica de pequena escala, as quais convertem diretamente a radiação solar em eletricidade. Já as tecnologias recentes são representadas por energia solar concentrada, onde se utiliza superfícies espelhadas para concentrar energia do sol, a fim de aproveitá-la em processos térmicos.

Os concentradores solares são equipamentos de fácil construção e operação. Permitem a captação da energia solar que atinge o topo da estrutura mediante uma superfície espelhada que a reflete para uma região denominada de focal. Essa região focal é facilmente identificada a partir do conhecimento do tipo de curvatura que gerou a superfície reflexiva do concentrador solar (Prado et al., 2012). Segundo tais autores, a curvatura parabólica é a mais indicada para sistemas que fazem uso da reflexão, haja vista proporcionar um ponto focal.

Assim, quando determinado corpo é posto diretamente no ponto focal do concentrador solar, ele 


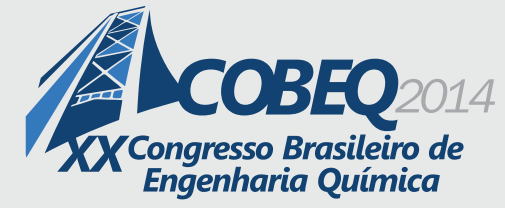

19 a 22 de outubro de 2014

Florianópolis/SC

é subitamente bombardeado pela radiação refletida, cujo efeito imediato é o rápido aumento da temperatura a patamares significativos, a depender da área disponível para reflexão, da radiação refletida e das dimensões do corpo de prova. Neste aspecto, este potencial térmico poderia ser perfeitamente aproveitado por muitos processos físico-químicos.

Isto posto, o presente trabalho teve como objetivo prever a temperatura alcançada por um corpo de prova quando inserido no ponto focal de um concentrador solar do tipo parabolóide de revolução. A previsão da temperatura a ser alcançada em cada situação foi obtida por simulação computacional mediante a manipulação da dimensão do coletor, da dimensão do corpo de prova e do fluxo de radiação incidente - fatores estes organizados sob a forma de um Planejamento Composto Central.

\section{METODOLOGIA}

Nas presentes simulações, partiu-se da premissa de que a superfície espelhada (parabolóide de revolução) tivesse como corte uma curvatura do tipo $\mathrm{f}(\mathrm{x})=\mathrm{ax}^{2}$ ou $\mathrm{g}(\mathrm{y})=(\mathrm{y} / \mathrm{a})^{1 / 2}$, com diâmetro de abertura de topo igual a $\mathrm{D}$. No que tange ao aquecimento do corpo de prova com dimensão característica "d", quando inserido no ponto focal do concentrador solar, o modelo fenomenológico que pode descrevê-lo está baseado em um Balanço de Energia, cujas principais contribuições estão esquematizadas na Figura 1.

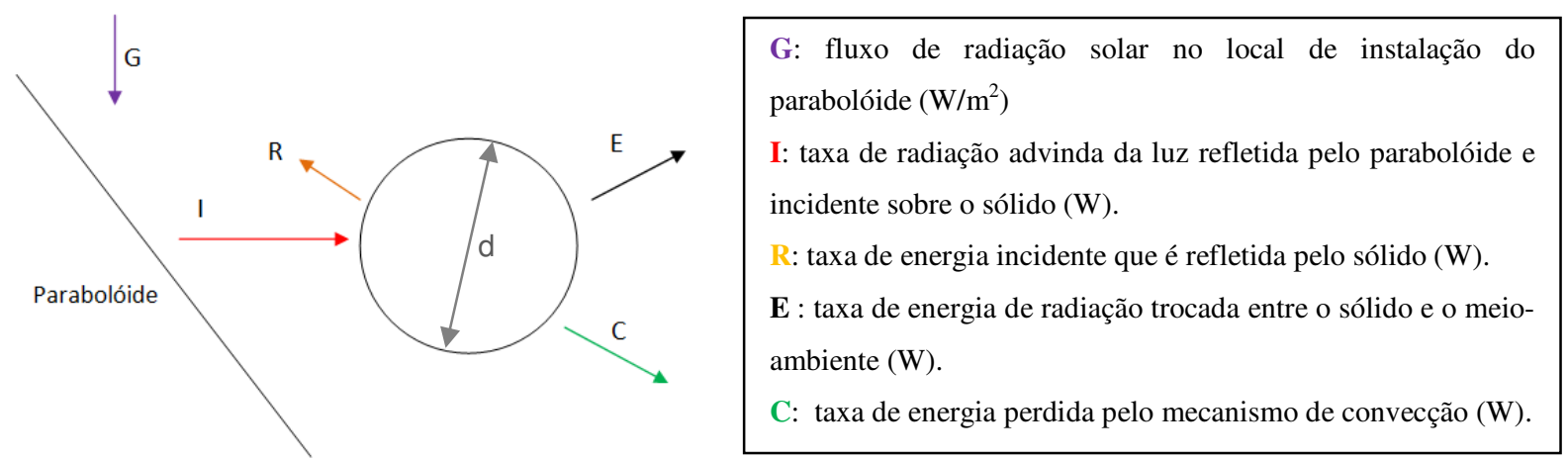

Figura 1 - Contribuições de calor responsáveis pelo aquecimento de um corpo de prova inserido no ponto focal de um concentrador solar

Em termos físico-matemáticos, as contribuições mencionadas na Figura 1 podem ser sistematizadas através do Balanço de Energia (efetuado no sólido) representado pela Equação 1.

$$
\rho \mathrm{Vc}_{\mathrm{p}} \frac{\mathrm{dT}}{\mathrm{dt}}=\mathrm{I}-\mathrm{R}-\mathrm{E}-\mathrm{C}
$$

Aplicando informações advindas dos Fenômenos de Transporte, os termos do segundo membro da Equação 1 podem ser reescritos, conforme mostra a Equação 2. 


$$
\rho \mathrm{Vc}_{\mathrm{p}} \frac{\mathrm{dT}}{\mathrm{dt}}=\rho_{\mathrm{P}}\left(\frac{\pi \mathrm{D}^{2}}{4} \mathrm{G}\right)-\rho_{\mathrm{C}}\left[\rho_{\mathrm{P}}\left(\frac{\pi \mathrm{D}^{2}}{4} \mathrm{G}\right)\right]-\varepsilon \mathrm{F}_{\mathrm{C} \rightarrow \mathrm{V}}\left(\pi \mathrm{d}^{2}\right) \sigma\left(\mathrm{T}^{4}-\mathrm{T}_{\infty}^{4}\right)-\mathrm{h}\left(\pi \mathrm{d}^{2}\right)\left(\mathrm{T}-\mathrm{T}_{\infty}\right)
$$

No equacionamento anterior, uma das principais dificuldades consiste no desconhecimento de algumas propriedades físicas do sistema, tais como a refletividade da superfície do parabolóide $\left(\rho_{\mathrm{P}}\right)$, a refletividade do corpo de prova $\left(\rho_{\mathrm{C}}\right)$, a emissividade do corpo de prova $(\varepsilon)$ e o fator de forma entre o corpo de prova com suas vizinhanças $\left(\mathrm{F}_{\mathrm{C} \rightarrow \mathrm{V}}\right)$. Todavia, a depender das condições operacionais utilizadas durante o processo de aquecimento, é possível contornar tal dificuldade. Por exemplo, em se tratando de superfícies refletoras (espelhos ou metais polidos) é bem provável que a refletividade geralmente tenda a $100 \%\left(\rho_{\mathrm{C}}=1\right)$. Por sua vez, o corpo de prova deixaria de refletir parte da energia incidente, absorvendo-a completamente, se e somente se, fosse considerado como negro. Claro que corpo negro é uma idealização física, mas que pode ser aproximada para determinados materiais (superfícies escuras, carbeto de silício etc.). $\mathrm{Na}$ hipótese de utilização destes materiais, certamente a refletividade e emissividade do corpo de prova tenderiam a 0 e $100 \%$, respectivamente $\left(\rho_{\mathrm{C}}=0\right.$ e $\varepsilon=$ 1). Por fim, como os concentradores solares são expostos ao meio-ambiente (lugares abertos e espaçosos), seria razoável considerar que o fator de forma do corpo a ser aquecido com sua vizinhança tenderia também à unidade $\left(\mathrm{F}_{\mathrm{C} \rightarrow \mathrm{V}}=1\right)$. Desta forma, considerando uma superfície polida, uma geometria esférica, um corpo negro e um ambiente de instalação "infinito", a Equação (2) pode ser reescrita sob a forma da Equação (3), cuja estrutura foi adotada para as simulações deste trabalho.

$$
\rho \mathrm{Vc}_{\mathrm{p}} \frac{\mathrm{dT}}{\mathrm{dt}}=\frac{\pi \mathrm{D}^{2}}{4} \mathrm{G}-\left(\pi \mathrm{d}^{2}\right) \sigma\left(\mathrm{T}^{4}-\mathrm{T}_{\infty}^{4}\right)-\mathrm{h}\left(\pi \mathrm{d}^{2}\right)\left(\mathrm{T}-\mathrm{T}_{\infty}\right)
$$

Em se tratando das perdas de calor por convecção, elas podem ocorrer de maneira forçada ou natural (Whitaker, 1972). Para tanto, a descrição do coeficiente convectivo de transferência de calor médio (h) utilizada neste trabalho (Incropera e DeWitt, 2003) foi baseada na Correlação de Churchill (convecção natural) e na Correlação de Whitaker (convecção forçada). Assim, a equação diferencial (Balanço de Energia - Equação 3) foi resolvida numericamente pelo Método de Euler (Ruggiero e Lopes, 1997), cujo passo de integração temporal $(\Delta t)$ foi de $1 \mathrm{~s}\left(\right.$ MAPLE $\left.^{\circledR}\right)$. Tratou-se de um problema de valor inicial, cuja condição de contorno foi $\mathrm{T}(0)=\mathrm{T}_{\infty}$. Desta forma, para cada passo de tempo, a temperatura $\left(\mathrm{T}_{\mathrm{i}}\right)$ foi estimada com base no valor da temperatura do passo anterior $\left(\mathrm{T}_{\mathrm{i}-1}\right)$, de acordo com a Equação 4.

$$
\mathrm{T}_{\mathrm{i}}=\mathrm{T}_{\mathrm{i}-1}+\frac{\Delta \mathrm{t}}{\rho \mathrm{Vcp}}\left[\frac{\pi \mathrm{D}^{2}}{4} \mathrm{G}-\sigma \pi \mathrm{d}^{2}\left(\mathrm{~T}_{\mathrm{i}-1}^{4}-\mathrm{T}_{\infty}^{4}\right)-\mathrm{h}_{\mathrm{i}-1} \pi \mathrm{d}^{2}\left(\mathrm{~T}_{\mathrm{i}-1}-\mathrm{T}_{\infty}\right)\right] \quad(1 \leq \mathrm{i}<\infty)
$$

Para a dinâmica de aquecimento, como referencial, foram considerados a densidade e o calor específico do carbeto de silício (aproximação de corpo negro) com valores de $2500 \mathrm{~kg} / \mathrm{m}^{3}$ e 700 $\mathrm{J} / \mathrm{kgK}$ (Incropera e DeWitt, 2003), respectivamente. Já a temperatura do fluido circundante ao sistema de aquecimento $\left(\mathrm{T}_{\infty}\right)$ foi de $20^{\circ} \mathrm{C}$. Nos casos de convecção forçada, foi adotada como velocidade média do fluido circundante ao sistema de aquecimento $\left(\mathrm{v}_{\infty}\right)$ o valor de $2 \mathrm{~m} / \mathrm{s}$, correspondente à média diária verificada na cidade de Uberlândia $(\mathrm{MG})$. Já a temperatura máxima $\left(\mathrm{T}_{\mathrm{M}}\right)$ a ser alcançada pelo corpo de prova durante o procedimento de aquecimento no concentrador solar foi determinada a partir da Equação 3, no momento em que todas as contribuições se compensavam (dT/dt $=0)$. 


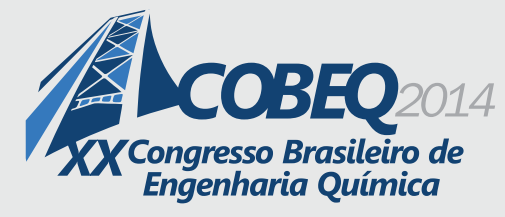

A fim de que as simulações computacionais pudessem ser realizadas de maneira sistemática, um Planejamento Composto Central (Box et al., 1978) foi concebido para estudar o comportamento da temperatura máxima $\left(\mathrm{T}_{\mathrm{M}}\right)$ a ser alcançada pelo corpo de prova mediante a manipulação do diâmetro do topo do parabolóide $[0,50 \leq \mathrm{D}(\mathrm{m}) \leq 3,00]$, do diâmetro do corpo de prova $[5 \leq \mathrm{d}(\mathrm{cm}) \leq 15]$ e da radiação solar do local de instalação $\left[250 \leq \mathrm{G}\left(\mathrm{W} / \mathrm{m}^{2}\right) \leq 850\right]$. Como esclarecimento, os valores mínimo e máximo atribuídos a $\mathrm{G}$ corresponderam às médias anuais de radiação na cidade de Uberlândia (MG), por volta das 10 e $13 \mathrm{~h}$, respectivamente. Os fatores $\mathrm{D}, \mathrm{d}$ e $\mathrm{G}$ foram codificados pelas Equações 5, 6 e 7, cuja nomenclatura adotada foi $X_{1}, X_{2}$ e $X_{3}$, respectivamente.

$$
\mathrm{X}_{1}=\frac{\mathrm{D}-1,75}{0,92}
$$

$$
X_{2}=\frac{d-10}{3,7}
$$

$$
\mathrm{X}_{3}=\frac{\mathrm{G}-550}{221}
$$

Consequentemente, a Matriz de Planejamento gerada para este trabalho, a partir dos fatores codificados (fator de ortogonalidade igual a 1,36), pode ser visualizada na Tabela 1.

Tabela 1 - Matriz de Planejamento para obtenção das Temperaturas Máximas $\left(\mathrm{T}_{\mathrm{M}}\right)$ a partir dos fatores codificados $(\alpha=1,36)$

\begin{tabular}{|c|c|c|c|c|c|c|c|c|c|c|c|}
\hline $\mathbf{N}$ & $\mathrm{X}_{1}$ & $\mathrm{X}_{2}$ & $\mathrm{X}_{3}$ & $\mathbf{N}$ & $\mathrm{X}_{1}$ & $\mathrm{X}_{2}$ & $\mathrm{X}_{3}$ & $\mathbf{N}$ & $\mathrm{X}_{1}$ & $\mathrm{X}_{2}$ & $\mathrm{X}_{3}$ \\
\hline $\mathbf{0 1}$ & -1 & -1 & -1 & $\mathbf{0 6}$ & +1 & -1 & +1 & $\mathbf{1 1}$ & 0 & $+\alpha$ & 0 \\
\hline $\mathbf{0 2}$ & -1 & -1 & +1 & $\mathbf{0 7}$ & +1 & +1 & -1 & $\mathbf{1 2}$ & 0 & $-\alpha$ & 0 \\
\hline $\mathbf{0 3}$ & -1 & +1 & -1 & $\mathbf{0 8}$ & +1 & +1 & +1 & $\mathbf{1 3}$ & 0 & 0 & $+\alpha$ \\
\hline $\mathbf{0 4}$ & -1 & +1 & +1 & $\mathbf{0 9}$ & $+\alpha$ & 0 & 0 & $\mathbf{1 4}$ & 0 & 0 & $-\alpha$ \\
\hline $\mathbf{0 5}$ & +1 & -1 & -1 & $\mathbf{1 0}$ & $-\alpha$ & 0 & 0 & $\mathbf{1 5}(\mathbf{2})$ & 0 & 0 & 0 \\
\hline
\end{tabular}

Por fim, para cada uma das linhas da Matriz de Planejamento, foi avaliada a máxima temperatura alcançada pelo corpo de prova (resposta), tanto para a convecção natural, quanto para a convecção forçada. De posse dessas 16 simulações, uma superfície de resposta para o tempo de aquecimento $\left(\mathrm{t}_{\mathrm{A}}\right)$ ou temperatura de equilíbrio $\left(\mathrm{T}_{\mathrm{M}}\right)$ - denominadas genericamente de $\mathrm{Y}$ - foi estimada por meio das Técnicas de Regressão Múltipla (STATISTICA ${ }^{\circledR} 7$ ), na qual os efeitos lineares, quadráticos e de interação foram apresentados sob a forma da Equação 8. Neste assunto, vale a ressalva de que os efeitos de interação da matriz quadrada são simétricos em relação à diagonal principal $\left(\mathrm{A}_{\mathrm{ij}}=\mathrm{A}_{\mathrm{ji}}\right)$ e que apenas os significativos (nível de significância menor ou igual a $5 \%$ ) foram mantidos na estrutura da Equação 8.

$$
Y=\beta+\left(\begin{array}{lll}
A_{1} & A_{2} & A_{3}
\end{array}\right)\left(\begin{array}{l}
X_{1} \\
X_{2} \\
X_{3}
\end{array}\right)+\left(\begin{array}{lll}
X_{1} & X_{2} & X_{3}
\end{array}\right)\left(\begin{array}{ccc}
A_{11} & \frac{A_{12}}{2} & \frac{A_{13}}{2} \\
\frac{A_{21}}{2} & A_{22} & \frac{A_{23}}{2} \\
\frac{A_{31}}{2} & \frac{A_{32}}{2} & A_{33}
\end{array}\right)\left(\begin{array}{l}
X_{1} \\
X_{2} \\
X_{3}
\end{array}\right)
$$

\section{RESULTADOS}

De acordo com as simulações realizadas, pôde-se perceber que os três fatores analisados diâmetro do topo do parabolóide (D), diâmetro do corpo de prova (d) e radiação (G) - foram importantes na dinâmica de aquecimento do corpo de prova, desempenhando cada qual seus efeitos 


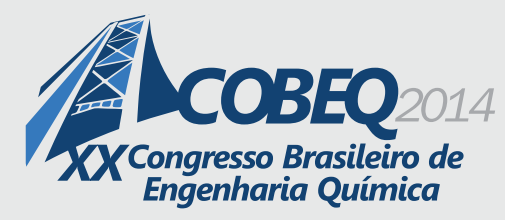

19 a 22 de outubro de 2014

Florianópolis/SC

específicos. A depender da combinação das condições operacionais utilizadas, a progressão temporal da temperatura pode se dar de maneira relativamente rápida ou demorada, até que a temperatura máxima (equilíbrio) seja alcançada. Para fins de ilustração, na Figura 2, são apresentadas as dinâmicas temporais da temperatura até que a temperatura de equilíbrio $\left(\mathrm{T}_{\mathrm{M}}\right)$ fosse alcançada (convecção natural). De acordo com as simulações efetuadas no caso de convecção natural (mesma tendência também verificada para convecção forçada), foi possível observar que as condições operacionais para as simulações 5, 6, 9 e 12 atingiram temperaturas de equilíbrio superiores a $900^{\circ} \mathrm{C}$ em menos de 10 min de aquecimento solar (Figura 2-a). Já as simulações 1, 2, 8, 13 e 15 foram capazes de atingir temperaturas de equilíbrio entre 400 e $800^{\circ} \mathrm{C}$ para tempos de aquecimento solar entre 10 e 20 min de aquecimento solar (Figura 2-b). Por sua vez, as simulações 3, 4, 7, 10, 11 e 14 levaram mais do que 20 min para poderem atingir temperaturas de equilíbrio inferiores a $500^{\circ} \mathrm{C}$ (Figura 2-c).
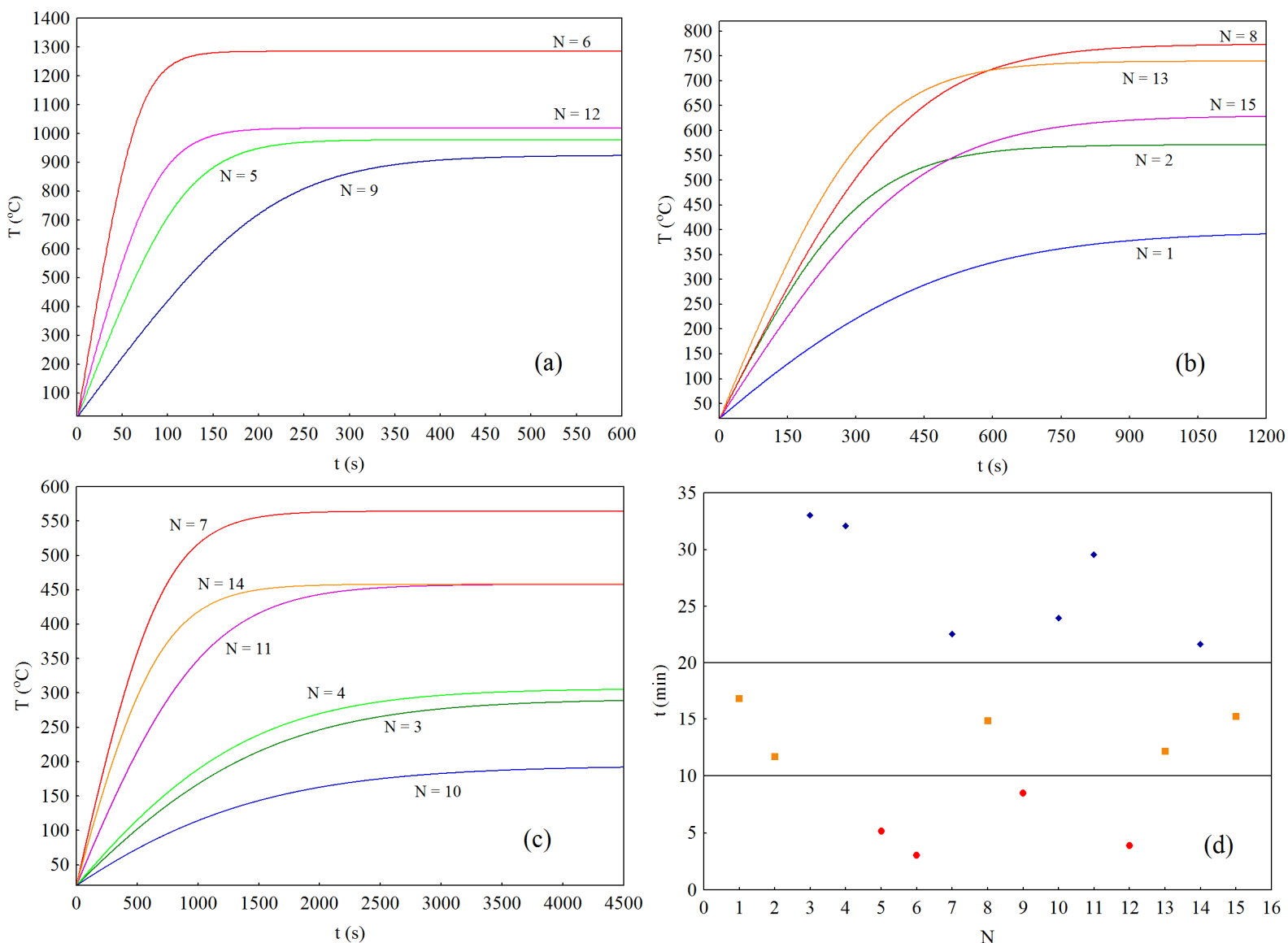

Figura 2 - Curvas de Aquecimento (a, b, c) e seus respectivos tempos de aquecimento (d).

A Equação da Superfície de Resposta estimada para o tempo de aquecimento, até que a temperatura de equilíbrio $\left(T_{M}\right)$ fosse alcançada, está representada pela Equação $9\left(R^{2}=0,9715\right)$, cujas informações advieram da Figura 2-d (síntese das Figuras 2-a,b,c). 


$$
\mathrm{t}_{\mathrm{A}}=16,83+\left(\begin{array}{lll}
-5,92 & 8,61 & -2,46
\end{array}\right)\left(\begin{array}{l}
\mathrm{X}_{1} \\
\mathrm{X}_{2} \\
\mathrm{X}_{3}
\end{array}\right)
$$

De acordo com a Equação 9, foi possível constatar que os fatores D, d e G exerceram forte efeito no tempo de aquecimento por energia solar. Constatou-se que os três fatores analisados $(\mathrm{D}, \mathrm{d}$ e G) desempenham apenas os efeitos lineares sobre o tempo de aquecimento, estando ausente, portanto, os efeitos de interação e os efeitos quadráticos. Dentre eles, restou esclarecido que o tamanho do sistema a ser aquecido (d) foi o efeito mais relevante sobre o tempo de aquecimento (efeito direto), seguido do tamanho do coletor solar (D) e do fluxo de radiação $(G)$, nesta ordem (efeitos inversos). Tal comportamento, explica o motivo pelos quais as simulações da Figura 2-a requisitaram um menor tempo de aquecimento dentre as demais, haja vista terem sido favorecidas pelos menores diâmetros do corpo de prova (d), pelos maiores diâmetros do coletor (D) e pelas maiores radiações de exposição $(\mathrm{G})$.

$\mathrm{Na}$ Figura 3, são apresentadas as máximas temperaturas $\left(\mathrm{T}_{\mathrm{M}}\right)$ alcançadas pelo concentrador solar (convecção natural e forçada), bem como a diferença térmica proporcionada pelos dois mecanismos convectivos de transferência de calor.
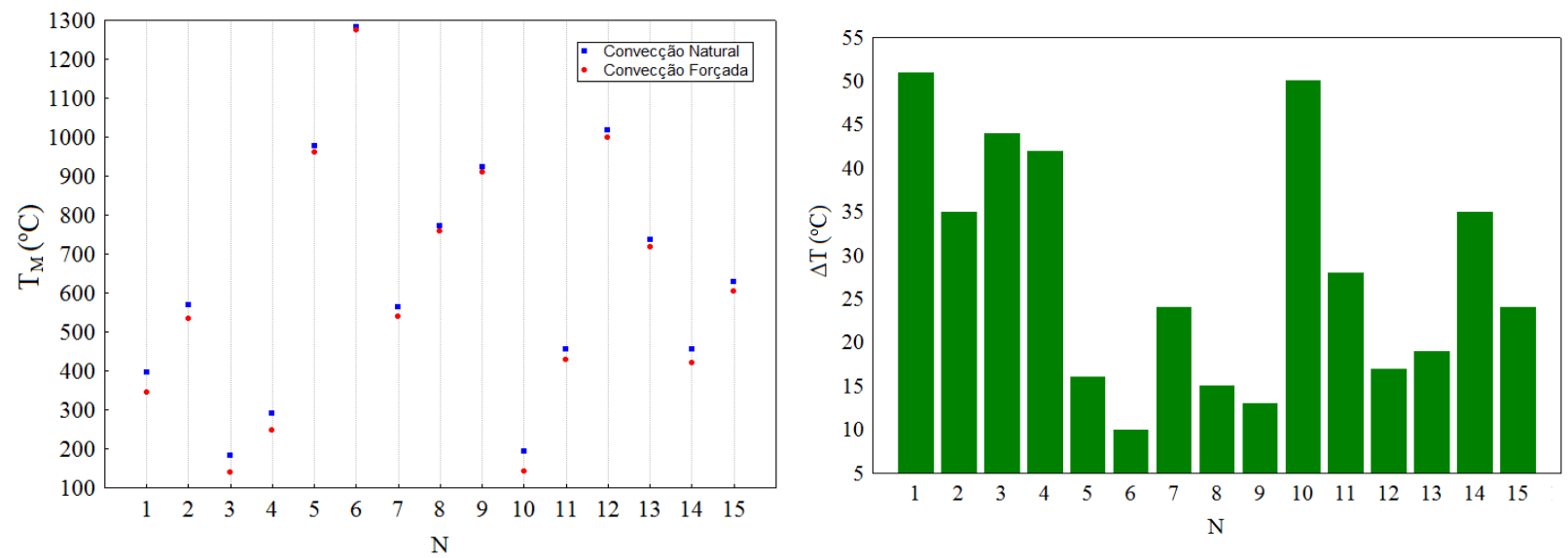

Figura 3 - Temperaturas máximas alcançadas pelo concentrador solar (a) e gradiente térmico entre convecção natural e forçada (b).

Como já era esperado, as simulações vieram a ratificar o fato de que as perdas de calor por convecção natural são menores do que na convecção forçada, o que implica, na primeira hipótese, na obtenção de maiores temperaturas de equilíbrio. Em média, as temperaturas máximas alcançadas no aquecimento solar sob condições de convecção natural foram aproximadamente $8 \%$ maiores do que nas condições de convecção forçada. Da mesma forma que fora realizado para o tempo de aquecimento $\left(t_{\mathrm{A}}\right)$, regressões múltiplas foram aplicas ao conjunto de respostas para a Temperatura Máxima de Aquecimento $\left(\mathrm{T}_{\mathrm{M}}\right)$, tanto para convecção natural quanto forçada, representadas respectivamente pelas Equações 10 e 11. 


$$
\begin{aligned}
& \mathrm{T}_{\mathrm{M}}=608,9+\left(\begin{array}{lll}
269,9 & -186,5 & 101,1
\end{array}\right)\left(\begin{array}{l}
\mathrm{X}_{1} \\
\mathrm{X}_{2} \\
\mathrm{X}_{3}
\end{array}\right)+\left(\begin{array}{ll}
\mathrm{X}_{1} \mathrm{X}_{2} \mathrm{X}_{3}
\end{array}\right)\left(\begin{array}{ccc}
-35,4 & -27,1 & 14,7 \\
-27,1 & 62,1 & 0 \\
14,7 & 0 & 0
\end{array}\right)\left(\begin{array}{l}
\mathrm{X}_{1} \\
\mathrm{X}_{2} \\
\mathrm{X}_{3}
\end{array}\right) \\
& \mathrm{T}_{\mathrm{M}}=546,7+\left(\begin{array}{lll}
283,4 & -188,9 & 105,8
\end{array}\right)\left(\begin{array}{l}
\mathrm{X}_{1} \\
\mathrm{X}_{2} \\
\mathrm{X}_{3}
\end{array}\right)+\left(\begin{array}{lll}
\mathrm{X}_{1} \mathrm{X}_{2} \mathrm{X}_{3}
\end{array}\right)\left(\begin{array}{ccc}
0 & -27,9 & 0 \\
-27,9 & 70,5 & 0 \\
0 & 0 & 0
\end{array}\right)\left(\begin{array}{l}
\mathrm{X}_{1} \\
\mathrm{X}_{2} \\
\mathrm{X}_{3}
\end{array}\right)
\end{aligned}
$$

Em se tratando das temperaturas de equilíbrio, não só os efeitos lineares foram significativos, como também os efeitos quadráticos e de interação foram relevantes. Neste tipo de resposta, o efeito mais relevante foi o diâmetro do topo do coletor solar (efeito direto), seguido pela dimensão do corpo de prova (efeito inverso) e pela radiação solar incidente (efeito direto), nesta ordem. Eis então, o motivo pelo qual, dentre as simulações realizadas, a $6^{\text {a }}$ simulação do PCC apresentou a maior temperatura de equilíbrio $\left(1284^{\circ} \mathrm{C}\right)$, haja vista que foi uma condição operacional que compatibilizou valores relativamente grandes de $\mathrm{D}$ e $\mathrm{G}$ com valores relativamente pequenos de diâmetro do corpo de prova (d). Em sentido contrário à simulação 6, ou seja, menores D e G combinados com maior d, foram observados os menores gradientes de temperatura para as simulações 3 e 10 que apresentaram temperaturas máximas de apenas 183 e $193^{\circ} \mathrm{C}$, respectivamente.

De acordo com os principais resultados deste trabalho, foi possível constatar que o aquecimento com energia solar pode proporcionar gradientes significativos de temperatura, os quais podem ser viáveis para serem utilizados no aquecimento de materiais ou transformações químicas (reações endotérmicas).

\section{CONCLUSÕES}

De acordo com os principais resultados obtidos neste trabalho, foi possível verificar que, a depender da combinação do tamanho do parabolóide (D), do corpo a ser aquecido (d) e da radiação solar incidente $(\mathrm{G})$, é possível obter altos patamares de temperatura para aplicações em diferentes demandas industriais. Na prática, sabe-se que as maiores temperaturas devem ser obtidas a partir dos menores tempos de aquecimento disponíveis. Para tanto, considerando uma determinada massa a ser aquecida, foi possível concluir que, quanto maiores forem a área disponível à coleta da luz e a intensidade desta radiação solar, maiores serão também as temperaturas máximas do sistema. Neste caso, sugere-se o uso dos maiores parabolóides possíveis e a captação de luz no período de maior intensidade de radiação solar (das 10 às 15 h). Foi possível também verificar que, além destas condições operacionais favoráveis, seria prudente manter o sistema de aquecimento protegido de ventos, haja vista que, sob condições de convecção natural, a temperatura geralmente obtida foi, em média, $8 \%$ superior àquelas verificadas para as hipóteses de convecção forçada.

\section{NOMENCLATURA}

C - taxa de perda de calor por convecção (W)

$\mathrm{cp}$ - calor específico do corpo de prova $(\mathrm{J} / \mathrm{kgK})$ 


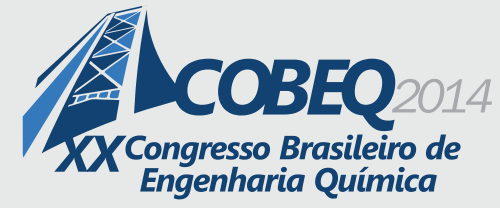

$\mathrm{d}$ - dimensão característica do corpo de prova $(\mathrm{cm})$

D - diâmetro do topo do parabolóide de revolução (m)

E - taxa de perda de calor por emissão (W)

$\mathrm{F}_{\mathrm{C} \rightarrow \mathrm{V}}$ - fator de forma entre o fator de forma e sua vizinhança (-)

$\mathrm{G}$ - fluxo de radiação incidente sobre o topo do paraboloide $\left(\mathrm{W} / \mathrm{m}^{2}\right)$

$\mathrm{h}$ - coeficiente convectivo de transferência de calor $\left(\mathrm{W} / \mathrm{m}^{20} \mathrm{C}\right)$

I - taxa de radiação refletida pelo parabolóide para o ponto focal (W)

$\mathrm{N}$ - numeração das linhas da matriz de planejamento (-)

$\mathrm{R}$ - taxa de radiação refletida pelo corpo de prova (W)

$\mathrm{V}$ - volume do corpo de prova $\left(\mathrm{m}^{3}\right)$

$\mathrm{t}_{\mathrm{A}}$ - tempo de aquecimento (s)

$\mathrm{T}$ - temperatura do corpo de prova $\left({ }^{\circ} \mathrm{C}\right)$

$\mathrm{T}_{\mathrm{M}}$ - temperatura máxima a ser atingida no concentrador solar pelo corpo de prova $\left({ }^{\circ} \mathrm{C}\right)$

$\mathrm{T}_{\infty}$ - temperatura ambiente $\left({ }^{\circ} \mathrm{C}\right)$

$\mathrm{X}_{1}, \mathrm{X}_{2}, \mathrm{X}_{3}$ - codificações para $\mathrm{D}, \mathrm{d}$ e $\mathrm{G}$, respectivamente (-)

$\varepsilon$ - emissividade da superfície do corpo de prova (-)

$\rho$ - densidade do corpo de prova $\left(\mathrm{kg} / \mathrm{m}^{3}\right)$

$\rho_{\mathrm{P}}-$ refletividade da superfície do parabolóide (-)

$\rho_{\mathrm{C}}-$ refletividade da superfície do parabolóide (-)

$\sigma$ - constante de Stefan-Boltzmann $\left(5,670 \times 10^{-8} \mathrm{~W} / \mathrm{m}^{2} \mathrm{~K}^{4}\right)$

\section{AGRADECIMENTOS}

Os autores deste trabalho agradecem à FAPEMIG (PCE-00082/2014 e CRA/APQ-01931/13), ao CNPq (Proj.422749/2013-7) e ao Laboratório de Separação e Energias Renováveis (LASER) da FEQUI/UFU.

\section{REFERÊNCIAS BIBLIOGRÁFICAS}

BOX, M.J.; HUNTER, W. G.; HUNTER, J. S.Statistics for experiments: na introdution to design, data analysis and model building, John Wiley and Sons, New York, 1978.

INCROPERA, F. P.; DEWITT, D. P. Transferência de Calor e Massa, $5^{a}$ Edição, Editora. LTC, 698 p., 2003.

NARBEL,P. A. ; TIMILSINA ,G. R.; KURDGELASHVIL, L. A Review of Solar Energy Market, Economics and Policies, Policy Research Working Paper 5845,2011.

PRADO, G. O.; AROUCA, A. M. M.; VIEIRA, L. G. M.; DAMASCENO, J. J. R. Utilization of Solar Energy as Heat Source. Materials Science Forum, v. 727, p. 114-118, 2012.

RUGGIERO, G. M.; LOPES R. V. Cálculo Numérico - Aspectos Teóricos e Computacionais, $2^{a}$ edição, Ed. Pearson, 1997.

WHITAKER, S. Forced convection heat transfer correlation for flow in pipes, past flat plates, single cylinders, single spheres and flow in packed beds and tube bundles, J. AICHE, 18, 361-371, 1972. 